\title{
Duodenal Web
}

National Cancer Institute

\section{Source}

National Cancer Institute. Duodenal Web. NCI Thesaurus. Code C98918.

Partial or complete obstruction of the duodenal lumen due to the presence of a membranous web. 